\title{
The effects of illumination level and retinal size on the apparent strength of subjective contours
}

\author{
SUSAN T. DUMAIS and DRAKE R. BRADLEY \\ Bates College, Lewiston, Maine 04240
}

\begin{abstract}
The apparent strength of subjective contours was investigated as a function of illumination level, figure size, and viewing distance. Magnitude estimation, with a real contour standard as the modulus, was used to measure the perceived strength of the illusory contours. It was found that illumination level and retinal size are both powerful determinants of the apparent strength of subjective contours, generating magnitude estimates varying from $20 \%$ to $96 \%$ of the strength of the real contour modulus. Particularly strong subjective contours were reported for figures of small retinal size (1.2 to $4.8 \mathrm{deg})$ under very dim illumination $(.10 \log \mathrm{lx})$.
\end{abstract}

Normally, a contour is defined as the border separating nonhomogeneous regions in the visual field. The stimulus conditions giving rise to such contours are usually abrupt changes in luminance, wavelength, or purity between adjacent regions in the stimulus display. Schumann (1904) first reported the appearance of illusory contours, ${ }^{1}$ such as those seen in Figure 1a, which extend over objectively homogeneous regions of the visual display. In the central region of this configuration some observers report seeing a whiter-than-white square bounded on the left and right sides by faint contours extending between the top and bottom segments of the black bordering region. These illusory contours are rather weak and unstable, especially when the figure subtends a large visual angle and is viewed under conditions of fixation. Schumann postulated that these contours result from an organizational process similar to the Gestalt principle of closure. Kanizsa (1955) extended Schumann's work in this area, and since then a number of configurations have been presented in which stable and salient subjective contours are seen by most observers (Figures $1 \mathrm{~b}$ and $1 \mathrm{c}$ ). For example, in Figure 1b, very compelling subjective contours corresponding to the "sides" of a triangle can be seen extending between the black inducing elements. In this and other subjective contour figures (see Kanizsa, 1974), a phenomenally complete object of uniform appearance, brighter or more intense than its background, displaced in the third dimension, and delineated by subjective edges or contours, is present for most observers. Although there is general agreement as to the consistent association of these phenomena in subjective contour configurations, ${ }^{2}$ there is nothing in this observation suggesting a particular causal chain of events.

We wish to ack nowledge the support of this research by a Faculty Research Grant (837-00-Bradley) awarded to D.R.B. by Bates College. S.T.D. is currently at Indiana University. Requests for reprints should be sent to Susan Dumais, Department of Psychology, Indiana University, Bloomington, Indiana 47401.
In addition to an experienced phenomenal reality, subjective contours and the objects delineated by them have a strong functional reality in that they can cause and undergo visual masking, optical illusions, tilt aftereffects, binocular rivalry, and figural aftereffects in much the same way as real contours and objects (Farné, 1968; Goldstein \& Weintraub, 1972; Gregory, 1972; Harris \& Gregory, 1973; Kanizsa, 1974; Pastore, 1971; Smith \& Over, 1975; Stadler \& Dieker, 1969, 1972; Weisstein, Matthews, \& Berbaum, Note 4). Furthermore, when identical stimuli are superimposed on the apparently closer subjective contour object and on the apparently more distant background (Figure 1), apparent size distortions are obtained in the direction predicted by a size-distance invariance relation (Coren, 1972). Finally, a larger brightness increment threshold is

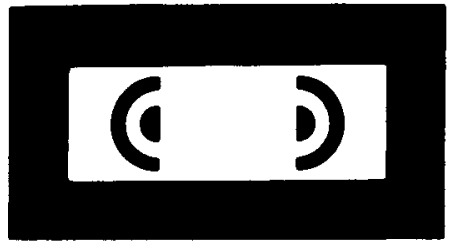

(o)

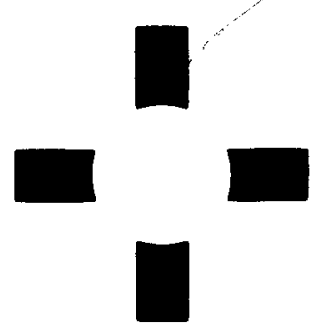

(c)

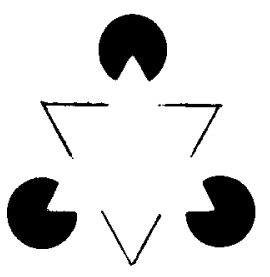

(b)

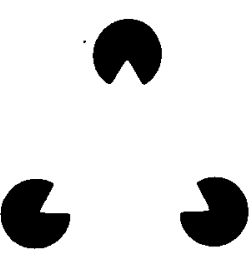

a
Figure 1. Subjective contour configurations: (a) the original Schumann (1904) figure, (b) a figure from Kanizsa (1955) in which salient subjective contours corresponding to the sides of a triangle are seen by most observers, (c) a figure demonstrating that curvilinear subjective contours can be produced, and (d) the configuration used in the present experiment. 
obtained when measured over the apparently brighter subjective contour object than over its background (Coren \& Theodor, Note 3), a finding consistent with the psychophysics of real brightness differences.

Since Schumann's original "closure" hypothesis, a number of theoretical explanations have been proposed to account for the subjective contour phenomenon. Kanizsa $(1955,1974)$ suggests that a tendency toward "amodal" completion of the inducing elements results in the stratification of the visual field into a surface and a background, situated at different levels, and separated by subjective contours. Coren (1972) argues that the processing of implicit depth cues (e.g., interposition cues in Figure 1) results in the creation of a plane or surface that is stratitied in depth relative to the rest of the array. As with Kanizsa, subjective contours are merely the edges or boundaries of this plane. In a yet more cognitive vein, Gregory (1972) claims that subjective contours are the boundaries of objects which are postulated ("object hypotheses") in order to account for blank sectors in the inducing elements. Bradley and Dumais (Note 1) suggest that subjective contours are simply the phenomenal correlates of the "edges" of visually constructed objects as implied by an analysis-by-synthesis theory of form perception (Neisser. 1967). In each of these theories, the observer plays an active role in the perceptual process by going bevond the sensory information present in the stimulus array in order to arrive at the final percept.

With regard to possible physiological mechanisms, Stadler and Dieker (1969. 1972) argue that subjective contours arise from the stimulation of partially activated contour detectors. Similarly, Jung and Spillman (1970) postulate that interactions among the receptive fields of orientation-specific cortical cells give rise to subjective contours. Higher order neural structures such as those proposed by Hebb (1949) point to another possible physiological basis for the processes involved in the perception of subjective contours.

Although the preceding theories have been fairly successful in accounting for the presence of objects. contours, and displacement in the third dimension in subjective contour displays, they generally fail to explain the consistently observed brightness differences. Bradley and Dumais (1975) argue that the apparent brightness difference between a subjective contour object and its background is simply a manitestation of a classic figure-ground phenomenon: i.e.. figures generally appear brighter or more intense than a background of equal reflectance value (Rubin, 1915). Alternative theories consider the apparent brightness difference as the primary phenomenon and derive subjective contours. objects. and stratification from this more fundamental process. For example, Kennedy (1975) suggests that apparent reflectance differences which arise as the result of a central attentional process can account for monocular subjective contours. Brigner and Gallagher (1974) argue that the black inducing elements in Figure 1 produce brightness induction of the central white regions of the displays. These areas of enhanced brightness result in the impression of an overlaying whiter-than-white object bounded by subjective contours at the points of transition in apparent brightness.

It is not, however, clear how simultaneous brightness contrast can explain the specific shape and homogeneous appearance of the subjectively bounded objects. or deal with often cited counterexamples where observations do not agree with predictions made on the basis of this theory (e.g., Coren \& Theodor. 1975; Kanizsa, 1974; Varin, 1971). Furthermore, Bradley and Petry (Note 2) and Bradley and Dumais (1975. Note 1) present subjective contour configurations which are ambiguous with respect to where the subjective contours can be seen. They note that the subjective contours and apparent brightness differences can shift locations or disappear entirely depending on the perceived organization of the ambiguous array. None of the above theories can. without extension. accommodate this finding.

It is perhaps surprising that, with the plethora of theories available to explain subjective contours, so little quantitative or parametric research has been conducted on this phenomenon. In part, this is a result of the lack of proper methodological procedures for measuring the apparent strength of 'subjective contours. In the following research, an attempt has been made to develop such a procedure in an experiment where several variables were parametrically investigated to determine their effect on the perceived strength of subjective contours. The simplified configuration presented in Figure 1d was used in the experiment. In order to establish a quantitative index of subjective contour strength based on a known standard and amenable to parametric statistical analysis, a variation of the magnitude estimation procedure (Stevens, 1956) was employed, using a real contour standard as the modulus.

Quantitative measures of the perceived strength of subjective contours as a function of figure size. viewing distance, and illumination level were obtained. From informal observations made by Schumann (1904), Coren (1972), and Kanizsa (1955, 1974), one would expect apparent contour strength to vary inversely with visual angle. The nature of the function relating visual angle and apparent contour strength is, however, unknown. Furthermore, since an infinite number of figure size/distance combinations can generate the same visual angle, the above observations are ambiguous and incomplete. If retinal size is the important factor, one would predict that at a given distance the smallest figure size would 
generate the most salient subjective contours. Similarly, for a given figure size, the strongest contours should be achieved at the greatest distance. However, if physical size and/or distance give rise to effects other than those mediated by retinal size, then differences in the perceived strength of subjective contours should be observed when different figure size/distance combinations are used to generate the same visual angle.

lllumination level was examined because while conducting informal investigations it was noted that the perceived strength of subjective contours seemed to vary with changes in illumination. Theories based on object hypotheses. depth cues, amodal completion, or closure make no obvious predictions with regard to this parameter. However, the results of physiological and experimental work have indicated that lateral inhibitory activity and apparent brightness contrast both increase with increases in illumination (Jameson \& Hurvich, 1961; Leibowitz, Myers, \& Chinetti, 1955; Ratliff. 1974). It follows that any subjective contour theory in which one or both of these mechanisms plays a major causal role (e.g., Brigner \& Gallagher, 1974) would predict increases in perceived contour strength with increases in illumination.

\section{METHOD}

\section{Subjects}

Thirty college undergraduates (15 males and 15 females), ranging in age from 17 to 21 , served as observers, in partial fulfillment of a course requirement.

\section{Design}

A 3 by 3 by 5 mixed factorial design with three figure sizes (10.16, 20.32 , and $40.64 \mathrm{~cm}$ "sides"), three viewing distances (121.92, 243.84, and $487.68 \mathrm{~cm})$, and five illumination levels $(.10,1.49$, $2.21,2.65$, and $2.89 \log 1 \mathrm{x}$ ) was employed. Viewing distance was the only between-groups factor. The particular figure sizes and distances selected generate five unique retinal sizes ranging from 1.2 to 18.9 deg visual angle, and such that the intermediate retinal sizes ( 2.4 to $9.5 \mathrm{deg}$ ) are achieved by two or more different figure size/distance combinations (Table 1). This design feature permits the effects of retinal size, physical size, and distance to be assessed individually. Ten observers were randomly assigned to each of the three distance conditions, where they viewed 15 randomly presented combinations of three figure sizes under five levels of incident illumination. These 15 combinations were again presented in a new random order providing a total of 30 trials. A magnitude estimation technique was used to obtain quantitative measures of the apparent strength of the subjective contours as compared to a real contour standard.

\section{Apparatus and Materials}

The subjective contour configurations (Figure 1d) were made by inking black inducing elements $(6.7 \%$ reflectance) on $53.34 \mathrm{x}$ $71.12 \mathrm{~cm}$ white poster board $(82 \%$ reflectance). Three different size subjective triangles were constructed using 10.16, 20.32 , and $40.64 \mathrm{~cm}$ "sides" and black inducing elements with $1.91,3.81$, and $7.62 \mathrm{~cm}$ radii, respectively. The ratio of size of inducing element to size of triangle was therefore held constant. In addition, a real contour triangle having $20.32-\mathrm{cm}$ sides was made by superimposing a white poster board triangle (of the same material as that used for the subjective contour stimuli) on three black disks $(3.81 \mathrm{~cm}$ radii) which were inked on $53.34 \times 71.12 \mathrm{~cm}$ white poster board. The reflectance values of the white and black areas of the real contour standard were the same as those of the subjective contour stimuli. Consequently, the only difference between the real and subjective contour stimuli was that in the former a real triangle (with clearly perceptible contours) was actually overlaying the disks, whereas in the latter a similar impression was created using specially notched and arranged inducing elements.

The subjective contour configurations were placed flush against a pale yellow wall at approximately eye level. They were illuminated by a 100 -W light bulb situated $91.44 \mathrm{~cm}$ from the display at an angle of $30 \mathrm{deg}$ from the perpendicular. A $135-\mathrm{V}$ variac controlled the level of illumination incident on the stimuli. (Illumination levels were measured using a Gossen Luna-Pro lightmeter calibtated for accuracy against a Spectra photometer, Model FC-200.) the real contour triangle was always situated $121.92 \mathrm{~cm}$ from the observer and $90 \mathrm{deg}$ to the right, again at eye level. This configuration was illuminated using a diffuse light source (a $30-\mathrm{W}$, "glow box" model $12-21 \mathrm{D})$ to minimize differential border shadows being cast by the superimposed triangle. The light source was $60.96 \mathrm{~cm}$ from the standard, and its intensity was constant at $.62 \log 1 \mathrm{x}$. Hence, the incident illumination, size, and distance of the real contour triangle were held constant in order to insure a constant modulus for comparison. To prevent scattered light from affecting the illumination level of the subjective contour displays, the diffuse light source and real contour triangle were enclosed on all but the side facing the observer.

\section{Procedure}

The illumination level was initially set at the intermediate value of $2.21 \log 1 \mathrm{x}$. The experimenter briefly explained the subjective contour phenomenon and the magnitude estimation technique. The observer was told that his task was to compare the apparent strength or salience of the subjective contours to the strength of the clearly perceptible, real contours of the standard. Three practice trials were given to provide the observer with some minimal experience at using magnitude estimation as a means of assessing the perceived strength of subjective contours. This procedure was adopted because it has been reported that the reliability of this psychophysical method is improved when an observer is provided with some degree of practice (Coren \& Girgus, 1972). A trial consisted of a 10-sec observation period, during which the observer inspected both the subjective and real contour displays. At the end of this period, the magnitude estimate of apparent contour strength was verbally reported. The observer was instructed to keep both eyes closed between trials while the figure size and/or illumination level of the subjective contour display were changed.

The experiment terminated with a postexperimental interview in which the observer was asked to comment on the subjective contour phenomenon, especially with regard to the conditions producing the strongest contours.

\section{RESULTS AND DISCUSSION}

Figure 2 presents the mean apparent strength of the subjective contours as a function of illumination level, viewing distance, and figure size. The distance conditions are coded by using identical data points,

Table 1

Visual Angle (Degrees) Generated by All Possible Figure Size/Distance Combinations

\begin{tabular}{crcc} 
& \multicolumn{3}{c}{ Viewing Distance $(\mathrm{cm})$} \\
Figure Size $(\mathrm{cm})$ & 121.92 & 243.84 & 487.68 \\
\hline 10.16 & 4.77 & 2.39 & 1.19 \\
20.32 & 9.53 & 4.77 & 2.39 \\
40.64 & 18.92 & 9.53 & 4.77 \\
\hline
\end{tabular}




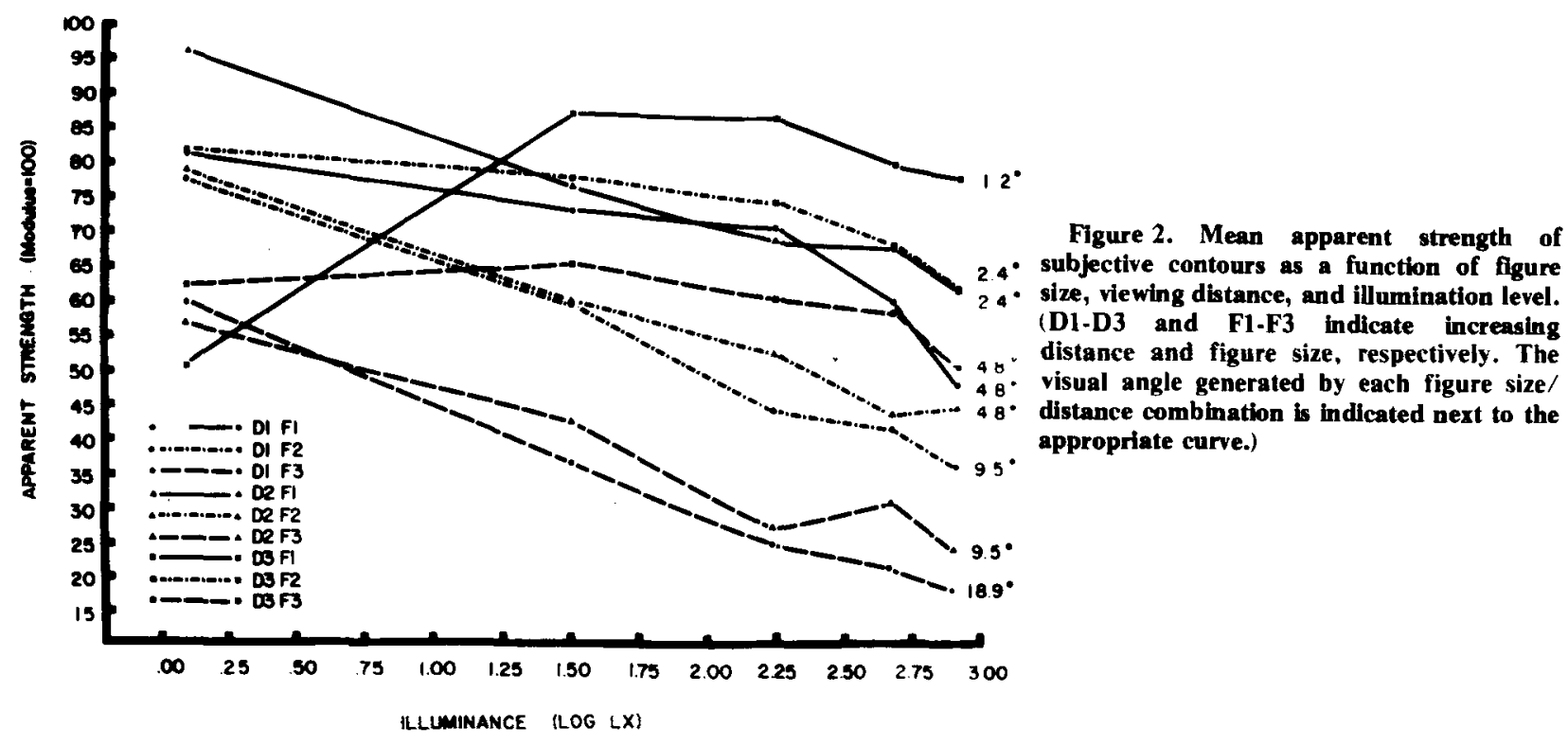

whereas the figure size conditions are coded by using identical connecting lines. The inset indicates the code for each unique combination of figure size and distance (F1-F3 and D1-D3 indicate increasing figure size and distance, respectively). In addition, the visual angle generated by each figure size/distance combination is indicated next to the appropriate curve. Inspection of Figure 2 and a three-way mixed model analysis of variance revealed several major trends in the data. First, apparent contour strength varied inversely with the log of the illumination level. and this effect was highly significant $[F(4,108)=$ $31.79, \mathrm{p}<.0001]$. The main effects of figure size and viewing distance were also significant or near significant $[\mathrm{F}(2.54)=78.37, \mathrm{p}<.0001$, and $\mathrm{F}(2,27)$ $=3.01, \mathrm{p}<.064]$, with strong subjective contours occurring in those conditions having small figures and/or large viewing distances.

Unfortunately, any simple interpretation of the data is precluded by the "aberrant" data point evident at the lowest illumination level of the D3F1 curve in Figure 2. The presence of this data point is probably responsible for the fact that all of the two-way interactions and the three-way interaction were significant ( $p$ ranging from .007 to .0001 ). When the data from the lowest illumination level were deleted from the three-way analysis of variance, none of the interactions approached significance ( $\mathrm{p}$ ranging from .57 to .16), although the main effects of illumination level, figure size, and viewing distance remained significant $[\mathrm{F}(3,81)=33.45, \mathrm{p}<.0001$; $\mathrm{F}(2.54)=85.63, \mathrm{p}<.0001 ;$ and $\mathrm{F}(2.27)=5.68$. $\mathrm{p}<.009]$. Furthermore, we have good reason to believe that the unusual decrease in apparent contour strength at the lowest illumination level of the D3F1 curve was artifactual. Several observers reported difficulty in seeing the inducing elements clearly in this particular condition (1.2 deg visual angle,
$.10 \log (\mathrm{x})$. If, in fact. visual acuity was not adequate in this condition, then apparent contour strength would diminish because of the lowered effectiveness of the inducing elements in generating the subjective contours. With this qualification, the data can be summarized as follows: apparent contour strength significantly increased with decreases in the size and illumination of the figure, and with increases in viewing distance. Mean magnitude estimates range from $96 \%$ of the real contour standard under optimal conditions to $20 \%$ under the least favorable conditions (Figure 2).

The finding that apparent contour strength varied with the inverse log of the incident illumination is of considerable theoretical import. Simultaneous brightness contrast theory (Brigner \& Gallagher, 1974) predicts that apparent contrast, and therefore apparent contour strength. should increase as the incident illumination increases. This prediction was clearly not supported by the present data, which showed a significant trend in exactly the opposite direction. Cognitively oriented theories do not seem to contain mechanisms which can easily predict or account for the effect of illumination on the apparent strength of subjective contours. However, it is possible that apparent contour strength diminishes at high levels of incident illumination because information is made available which serves to weaken the impression of an occluding object superimposed over the background and inducing elements. For example, the border shadows which are normally cast by an overlaying object under strong illumination are not present in the subjective contour display. Furthermore, under strong illumination the microtexture of the stimulus display is more clearly visible, and the homogeneous textured appearance of the white regions of the display might cause the "triangle" and its background to merge perceptibly, thus weakening 


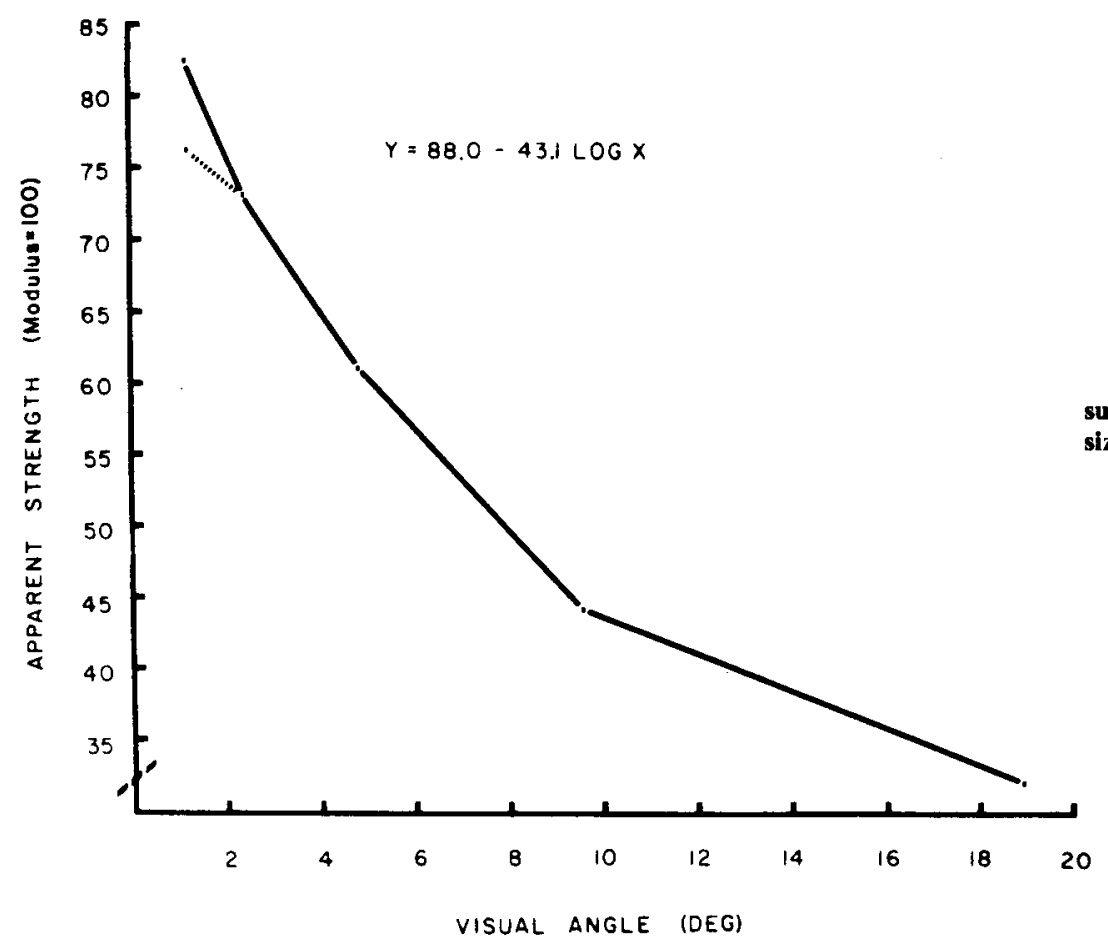

Figure 3. Mean apparent strength of subjective contours as a function of metinal size.

the subjective contours. Insofar as lowering the illumination level (within the limits of visual acuity) reduces what is objectively known about the stimulus array, one might expect an increase in the role of cognitive mechanisms such as Gregory's object hypothesis, and therefore an increase in the salience of the phenomena they generate.

If the effects of distance and figure size on the apparent strength of subjective contours are mediated simply by changes in retinal size, then an analysis of the relationship between the visual angle subtended by the figure and the apparent strength of the subjective contours should reveal a fairly systematic relationship. The data from "all experimental conditions giving rise to the same visual angle (if more than one) were pooled to obtain the data points plotted in Figure 3. The two data points over the 1.2-deg visual angle differentiate the case where the "aberrant" data point of Figure 2 was (lower point) and was not (upper point) included in the computation of mean apparent contour strength. An analysis of the best fitting function applied to the data of the solid curve revealed that an inverse log function $(Y=88.0-43.1 \log X)$ accounted for $99 \%$ of the variation in the data. ${ }^{3}$

The informal observation made by previous investigators (Coren, 1972; Kanizsa, 1955, 1974; Schumann, 1904) that subjective contours appear stronger in configurations which subtend small visual angles is certainly supported by the data presented in Figure 3. Retinal size is a powerful determinant of apparent contour strength regardless of whether changes in this variable are achieved by varying figure size, viewing distance, or both. It is important, however, to determine if figure size and distance exert additional influences on apparent contour strength which cannot be accounted for by differences in retinal size. A direct test of this possibility can be made by determining whether or not different figure size/distance combinations giving rise to the same visual angle result in significant differences in apparent contour strength. If retinal size alone is the critical factor, then significant differences should not be found. One-way analyses of variance were conducted for each of the visual angle conditions where two or more figure size/distance combinations gave rise to the same visual angle (Table 1). Mean apparent contour strength did not differ significantly across the three combinations giving rise to the 4.8-deg visual angle $[F(2.27)<1.0]$ or across the two combinations generating the 2.4-deg visual angle $[F(1,18)<1.0]$. A borderline significant difference was found, however, for the two combinations associated with the $9.5-\mathrm{deg}$ visual angle $[\mathrm{F}(1,18)=$ $4.21, \mathrm{p}<.053$ ]. We may conclude that retinal size per se is a critical parameter determining the apparent strength of subjective contours. If viewing distance and figure size have unique effects other than those mediated by retinal size, they are not detectable for the relatively small visual angles which are typically used to produce subjective contours (i.e., $4.8 \mathrm{deg}$ or less).

The theoretical significance of the inverse logarithmic relation between visual angle and apparent contour strength is not entirely clear, insofar as none of the theorics previously reviewed make explicit predictions regarding the effect of retinal size. A brightness contrast explanation (Brigner \& 
Gallagher. 1974) might argue that contrast is maximized for small visual angles since the retinal image is contined to the foveal region where the neural interactions giving rise to brightness contrast are strongest. Cognitively oriented theories such as Gregory.s (1972) might account for this data by assuming that the ability of an object hypothesis to support the impression of a phenomenally complete object is inversely proportional to the amount of "interpolation" required in the absence of sensory support. With large visual angles, subjective contours must be interpolated across large portions of the visual tield in order to implement the object hypothesis postulated from available sensory data. It is reasonable to suppose that the resulting percept would be weaker or less stable than one arising from a display where the visual angle of the completed object is quite small and the interpolation demand minimal. This same prediction follows if subjective contours are viewed as the result of some form of central facilitation based on the activity of partially activated cortical receptors (Stadler \& Dieker, 1969. 1972), interactions among receptive fields of orientationspecific cortical cells (Jung \& Spillman, 1970), or higher order neural structures (Hebb, 1949). As the interpolation demand increases with large visual angles, the central facilitation of the perception of a complete object should weaken.

In summary, the results of the present experiment indicate that extremely salient subjective contours can be created by viewing figures of small retinal size in dim illumination. Under these conditions, magnitude estimates range between $78 \%$ and $96 \%$ of the strength of clearly perceptible real contours viewed as the modulus. By varying illumination level over a range of nearly three log units and visual angle over a range of $17 \mathrm{deg}$. a wide range of magnitude estimates was obtained $(20 \%$ to $96 \%)$, indicating the potency of these variables in affecting the perceived strength of subjective contours. It is hoped that the application of magnitude estimation procedures will provide future investigators with a sensitive method for assessing the relative importance of other variables in affecting the perceived strength of subjective contours.

\section{REFERENCE NOTES}

1. Bradley. D. R.. \& Dumais. S. T. Simultaneous brightness contrast is. organizational determinants of subjective contours. Manuscript submitted for publication. 1975.

2. Bradley. D. R.. \& Petry, H. M. Organizational determinants of subiective contours: The subjective Necker cube. Manuscript submitted for publication. 1975.

3. Coren. S.. \& Theodor. L. Neural interactions and subjective contours. Manuscript submitted for publication. 1975.

4. Weisstein. N., Matthews, M.. \& Berbaum, K. Illusony contours can mask real contours. Paper presented at the meeting of the Psichomonic Siciets. Boston. November 1974.

\section{REFERENCES}

Bradley. D. R.. \& Dumais. S. T. Ambiguous cognitive contours. Nature. 1975. 257. 582.584.

Brigner. W. L.. \& Gallagher. M. B. Subjective contour: Apparent depth or simultaneous brightness contrast? Perceptual and Motor Skills, 1974, 38, 1047-1053.

Coren. S. Subjective contours and apparent depth. Psychological Review. 1972. 79. 359-367.

Coren. S.. \& Girgus. J. A comparison of tive methods of illusion measurement. Behavioral Research Methods \& Instrumentution, 1972, 4. 240-244.

Coren, S.. \& Theodor. L. Subjective contour: The inadequacy of brightness contrast as an explanation. Bulletin of the Psyhonomic Societr. 1975. 6. 87-89.

FARNÉ, M. Alcune osservazioni con linee virtuali e margini quasi percettivi. Bollkttino Società Italiana Biologia Sperimentale. 1968. 44. 1013-1610.

Goldstein. M. B.. \& Weintraub. D. J. The parallel-less Poggendorft: Virtual contours put the illusion down but not out. Perception \& Psychophysics. 1972, 11. 353-355.

Gregory. R. L. Cognitive contours. Nature. 1972. 238. $51-52$.

HARRIS. J. P.. d GREGORY. R. L. Fusion and rivalry of illusory contours. Perception. 1973. 2. 235-247.

HeBB, D. O. The organization of beharior. New York: Wiley, 1949.

JAMESON. D.. \& HuRvich, L. M. Complexities of perceived brightness. Silince. 1961. 133. 174-179.

Jung. R.. \& Spillman. L. Receptive-field estimation and perceptual integration in human vision. In F. A. Young \& D. B. Lindsley (Eds.). Early experience and visual intormation processing in perceptual and reading disorders. Washington. D.C: National Academy of Science, 1970.

Kanizsa. G. Marzini quasi-percettivi in campi con stimolazione omogenea. Rivista di Psicologia. 1955. 49. 7-30.

Kanizsa. G. Contours without gradients or cognitive contours? Italian Journal of Psichology, 1974. 1. 93-113.

KenNedY. J. M. Depth at an edge, coplanarity, slant depth. change in direction and change in brightness in the production of subjective contours. Italian Joumal of Psychology. 1975. 2. 107-123.

Lawson, R. B., \& Gulick. W. L. Stereopsis and anomalous contour. Vision Research, 1967, 7, 271-297.

Leibowitz. H. W.. Myers. N. A.. \& Chinetti. P. The role of simultaneous contrast in brightness constancy. Journal of Experimental Psychologi. 1955. 50. 15-18.

NeISSER, U. Cognitive psychology. New York: Appleton-CenturyCrofts, 1967.

OsGooD. C. E. Method and theon in experimental psuchology. New York: Oxford University Press. 1951.

PASTORE. N. Selective history of theories of visual perception 1650-1950. New York: Oxford University Press. 1971.

RatLiff. E. (Ed.). Studies on excitation and inhibition in the retina. New York: Rockefeller University Press. 1974.

Rubin. E. Sinsoplevede figurer. Copenhagen: Gyldendalske. 1915.

Schumann. F. Einige Beobachtungen über die Zusammenfassung von Gesichtseindrucken zu Einheiten. Psichologische Studien. 1904. 1. 1-32.

Sмith. A.. \& Over. R. Tilt aftereffects with subjective contours. Nature. 1975. 257. 581-582.

Stadler, M. . \& Dieker. J. Margini quasi-percettivi e after-effects tigurali. Rivista di Psicologia. 1969. 63. 95-104.

Stadler. M.. \& Dieker. J. Untersuchungen zum Problem virtueller Konturen in der visuellen Wahrnehmung. Zeitschrift fiir experime'ntelle und angewandte Psychologie. 1972. 19. $325-350$.

Stevens. S. S. The direct estimation of sensory magnitudesloudness. Americun Journal of Psychology. 1956. 69. 1-25.

Varin. D. Fenomeni di contrasto e diffusione cromatica nell organizazione spaziale del campo percettivo. Rivista di Psicologia. 14־1, 65. 101-128. 


\section{NOTES}

1. The term "subjective contour," introduced by Osgood (1951, p. 232), will be used to refer to these illusory contours. A variety of other terms, including anomalous contours (Lawson \& Gulick. 1967). cognitive contours (Gregory, 1972), quasi-perceptive margins (Kanizsa, 1955), and contours without gradients (Kanizsa, 1974), have also been used to describe the same phenomenon.

2. Kennedy (1975) has recently demonstrated that subjective contours which are typically associated with subjective impressions of depth, can also be found in the absence of apparent depth. He noted that subjective contours can be seen dividing two sheared regions which appear to be coplanar, and also that subjective contours corresponding to a corner formed by two planes meeting at dihedral angles can be observed.

3. A similar analysis of the curve terminating on the dashed line indicated that an inverse $\log$ function $(Y=83.8-38.9 \log X)$ accounted for $96 \%$ of the variation in the data of this curve.

(Received for publication September 10, 1975; revision received December 3, 1975.) 